\title{
Molecular analysis of the microflora in chronic venous leg ulceration
}

\author{
K. E. Hill, ${ }^{1}$ C. E. Davies, ${ }^{1}$ M. J. Wilson, ${ }^{1}$ P. Stephens, ${ }^{1}$ K. G. Harding ${ }^{2}$ \\ and D. W. Thomas ${ }^{1}$
}

\begin{abstract}
Correspondence
K. E. Hill

hillke1@cardiff.ac.uk
\end{abstract}

Received 25 July 2002

Accepted 18 October 2002

\section{Department of Oral Surgery, Medicine and Pathology ${ }^{1}$ and Wound Healing Research Unit, Department of Surgery², University of Wales College of Medicine, Cardiff CF14 4XY, UK}

\begin{abstract}
There is growing evidence to suggest that the resident microflora of chronic venous leg ulcers impairs cellular wound-healing responses, thereby playing an important role in maintaining the nonhealing phenotype of many of these wounds. The significance of individual species of bacteria will remain unclear until it is possible to characterize fully the microflora of such lesions. The limitations and biases of culture-based microbiology are being realized and the subsequent application of molecular methods is revealing greater diversity within mixed bacterial populations than that demonstrated by culture alone. To date, this approach has been limited to a small number of systems, including the oral microflora. Here, for the first time, the comprehensive characterization of the microflora present in the tissue of a chronic venous leg ulcer is described by the comparison of $16 \mathrm{~S}$ rDNA sequences amplified directly from the wound tissue with sequences obtained from bacteria that were isolated by culture. The molecular approach demonstrated significantly greater bacterial diversity than that revealed by culture. Furthermore, sequences were retrieved that may possibly represent novel species of bacteria. It is only by the comprehensive analysis of the wound microflora by both molecular and cultural methods that it will be possible to further our understanding of the role of bacteria in this important condition.
\end{abstract}

\section{INTRODUCTION}

Chronic venous leg ulcers (CVLU) affect approximately $1 \%$ of the UK population and are debilitating and painful. The aetiology of these wounds is not well understood, but it is likely to be multi-factorial. CVLU support a diverse microbial flora, and there is evidence that micro-organisms in this biofilm contribute to the non-healing phenotype. Moreover, these wounds occur in individuals and tissues that are at increased risk of bacterial invasion due to poor vascular supply and systemic factors (Falanga, 1993). Based as it is on an unclear understanding of the aetiology, management is

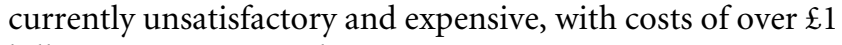
billion per annum in the UK.

Cultural analyses have documented that staphylococci, streptococci, enterococci and facultative Gram-negative bacilli are the bacterial groups most frequently recovered from CVLU (Hansson et al., 1995). However, more recently, the importance of fastidious and slow-growing species such as anaerobes is being established. With the employment of strict anaerobic isolation techniques and prolonged incubation times, almost $60 \%$ of CVLU have been shown to harbour anaerobic bacteria such as Fusobacterium spp. and

Abbreviation: CVLU, chronic venous leg ulcer. peptostreptococci (Halbert et al., 1992; Murdoch et al., 1994). However, it is becoming increasingly clear that there are considerable limitations to cultivation-dependent methods in determining the composition of complex microbial communities and that culture alone is unlikely to characterize fully the microflora present in chronic wounds. In a limited number of microbial systems, considerably greater diversity of the microflora has been revealed by molecular analysis, in particular the retrieval of rRNA gene sequences by PCR. In addition, molecular analysis is increasingly being employed as an adjunct to conventional cultural techniques in the analysis of diseases with complex microflora (Wilson et al., 1997). Sequences so retrieved are cloned and sequenced and the sequences compared to sequence databases for identification or phylogenetic characterization. For example, the study of the oral microflora associated with periodontal disease and purulent infection, in which it has been estimated that only $30-50 \%$ of microbial species are amenable to culture, has been significantly advanced by the application of such molecular tools (Kroes et al., 1999; Dymock et al., 1996).

In this study, we have applied both molecular and enhanced cultural techniques in the analysis of the microbial population within a single, chronic, non-healing, venous leg ulcer wound. 


\section{METHODS}

Patient details. Following ethical approval, a typical patient (i.e. elderly, obese, female) at the Wound Healing Research Unit, University Hospital of Wales, Cardiff, receiving standard compression therapy for a clinically non-infected wound, was selected for study with informed consent. Additional patient characteristics include: age, 59; height, 165 $\mathrm{cm}$; weight, $107 \mathrm{~kg}$; duration of wound, 2 years; wound size, $1310 \mathrm{~mm}^{2}$. There was no presence of systemic disease and the patient had not had systemic or topical antimicrobial therapy in the previous month.

Sampling of the wound. The wound surface was irrigated with sterile saline and the wound bed sampled via a surface swab and a $6 \mathrm{~mm}$ punch biopsy. The swab and tissue were transferred in separate vials of $(2 \mathrm{ml})$ reduced transport medium (TM) (Bowden \& Hardie, 1971) to the laboratory for processing within $1 \mathrm{~h}$ of collection. The tissue was divided aseptically into two portions using a sterile scalpel, one for cultural analysis and the other for molecular analysis.

Cultural analysis of wound microflora. The swab sample was vortexmixed in TM for $5 \mathrm{~min}$. The tissue for cultural analysis was cut up finely with a sterile scalpel and also vortex-mixed in TM for 5 min. Serial dilutions in TM were plated onto the following media (from LabM): blood agar (BA), fastidious anaerobe agar (FAA), both supplemented with $5 \%(\mathrm{v} / \mathrm{v})$ horse blood, MacConkey no. 3 and Sabouraud's medium. All plates except FAA were incubated aerobically at $37^{\circ} \mathrm{C}$. FAA plates were incubated under anaerobic conditions $\left(10 \% \mathrm{CO}_{2}\right.$, $10 \% \mathrm{H}_{2}, 80 \% \mathrm{~N}_{2}$ ) at $37^{\circ} \mathrm{C}$. Prolonged incubation of the macerated tissue was undertaken in fastidious anaerobe broth for 7 days prior to plating on FAA to allow recovery of fastidious and slow-growing anaerobic species. Primary isolation plates were initially examined after $48 \mathrm{~h}$ and then incubated for at least 10 days. Identification of bacteria followed standard microbiological schemes by examination of a range of phenotypic properties (staining reactions, colonial morphology, carbohydrate fermentation patterns) and, where appropriate, commercial identification kits. In this way, it was possible to identify each isolate to species level.

Molecular analysis of wound microflora. Molecular analysis was done on the tissue sample. DNA was extracted by standard techniques (Keay et al., 1998) with the addition of three freeze-thaw steps (each consisting of $2 \mathrm{~min}$ in liquid nitrogen and $2 \mathrm{~min}$ at $65^{\circ} \mathrm{C}$ ). DNA was extracted directly from the tissue sample and also from individual microbial species cultured from the wound. PCR was performed as described previously (Marchesi et al., 1998; Lane, 1991; Carroll et al., 2000; Klausegger et al., 1999) using three sets of universal primers. These were designated according to Escherichia coli $16 \mathrm{~S}$ rDNA numbering as follows: 63f/1387r (Marchesi et al., 1998), 27f/1492r and 27f/1525r (Lane, 1991). Two sets of primers designed primarily to amplify Grampositive bacterial species were also used in a nested PCR using primers 27f/1492r in the first round of PCR followed by a second PCR with primers 712f/1067r (Carroll et al., 2000) and 1185f/1540r (Klausegger et al., 1999). PCR was followed by ligation into the pCR2.1 TOPO vector (Invitrogen) and transformation into Top 10 competent Escherichia coli cells (Invitrogen). Blue/white screening of transformants was done on Luria-Bertani agar (Sambrook et al., 1989) containing $50 \mathrm{mg}$ ampicillin $\mathrm{ml}^{-1}$ and top-spread with $40 \mathrm{ml} \mathrm{X-Gal} \mathrm{solution}\left(20 \mathrm{mg} \mathrm{ml}^{-1}\right)$. Clones amplified from tissue were screened by RFLP analysis of M13-amplified PCR products using the restriction enzymes $C f o \mathrm{I}, \mathrm{RsaI}$ and HaeIII (Promega). DNA for sequencing was prepared from clones using Wizard Plus SV minipreps (Promega). Clones with unique RFLP patterns and two clones from each cultured organism were sequenced and both strands were sequenced on an automated laser fluorescence sequencer (ABI 377; PE Applied Biosystems) by using primers M13f and M13r, giving double coverage of $>1 \mathrm{kbp}$ of $16 \mathrm{~S}$ rDNA for the universal primer products for phylogenetic analysis. Gram-positive primer products were considerably smaller, 355 and 365 bp (Carroll et al., 2000; Klausegger et al., 1999), for which $100 \%$ double coverage was achieved. Sequences obtained were compared to database sequences and phylogenetic trees were constructed as described previously (Hill et al., 1999).

\section{RESULTS}

Cultural analysis revealed that Acinetobacter spp. and Staphylococcus epidermidis were found in large numbers (respectively $6.0 \times 10^{4}$ and $2.0 \times 10^{4}$ c.f.u. $\mathrm{ml}^{-1}$; Table 1$)$ in the wound tissue and that no additional cultural isolates were obtained after prolonged anaerobic incubation of the sample. Distinct differences were observed between sample sites (Table 1). Two species, Staphylococcus epidermidis and Proteus spp., were isolated from the tissue and the swab, respectively, but not from both sample sites, and Acinetobacter spp. were found in large numbers in both the tissue and the swab.

The results of the molecular analysis are shown in the dendrogram in Fig. 1 and in Table 2. In total, 26 clones amplified using five different primer sets were sequenced. It was possible by PCR to amplify sequences from bacteria found in the tissue and, in addition, sequences representing bacteria cultured from the swab only, e.g. Proteus spp., were detected. Fig. 1 shows that the sequences retrieved varied to some extent with the primer pairs used. For example, clones 3_3 and 3_1, showing greatest similarity to Acinetobacter haemolyticus and Bacteroides ureolyticus, respectively, were only amplified using primers $27 f / 1525$ r. Furthermore, other clones obtained using the other two universal primer pairs were not found in this part of the tree. Likewise, Grampositive species were only amplified when using the Grampositive-specific primers $712 \mathrm{f} / 1067 \mathrm{r}$ and $1185 \mathrm{f} / 1540 \mathrm{r}$ in a nested PCR and not when using any of the three universal primer sets (Table 2). The latter sequences were unsuitable for inclusion in the dendrogram due to a lack of overlap between the two primer pairs and also because of their short length ( 355 and $365 \mathrm{bp}$ ), which would have compromised the robustness of the tree.

All of the sequences recovered from bacteria cultivated from the wound tissue were $>98 \%$ identical to sequences within the public databases. In contrast, the 16S rRNA sequences recovered by direct amplification from tissue showed a greater degree of variability. For example, clones of the amplified sequences in cluster A, namely clones $0 \_16,0 \_11$ and $0 \_10$, showed more than $5 \%$ dissimilarity from any

Table 1. Cultural analysis of swab and tissue samples

Values are c.f.u. $\mathrm{ml}^{-1}$. ND, Not detected.

\begin{tabular}{|lcc|}
\hline Species & Swab & Tissue \\
\hline Acinetobacter spp. & $5.48 \times 10^{6}$ & $6 \cdot 0 \times 10^{4}$ \\
Staphylococcus epidermidis & $\mathrm{ND}$ & $2 \cdot 0 \times 10^{4}$ \\
Proteus spp. & $3.6 \times 10^{6}$ & $\mathrm{ND}$ \\
Candida tropicalis (yeast) & 4 & $\mathrm{ND}$ \\
\hline
\end{tabular}




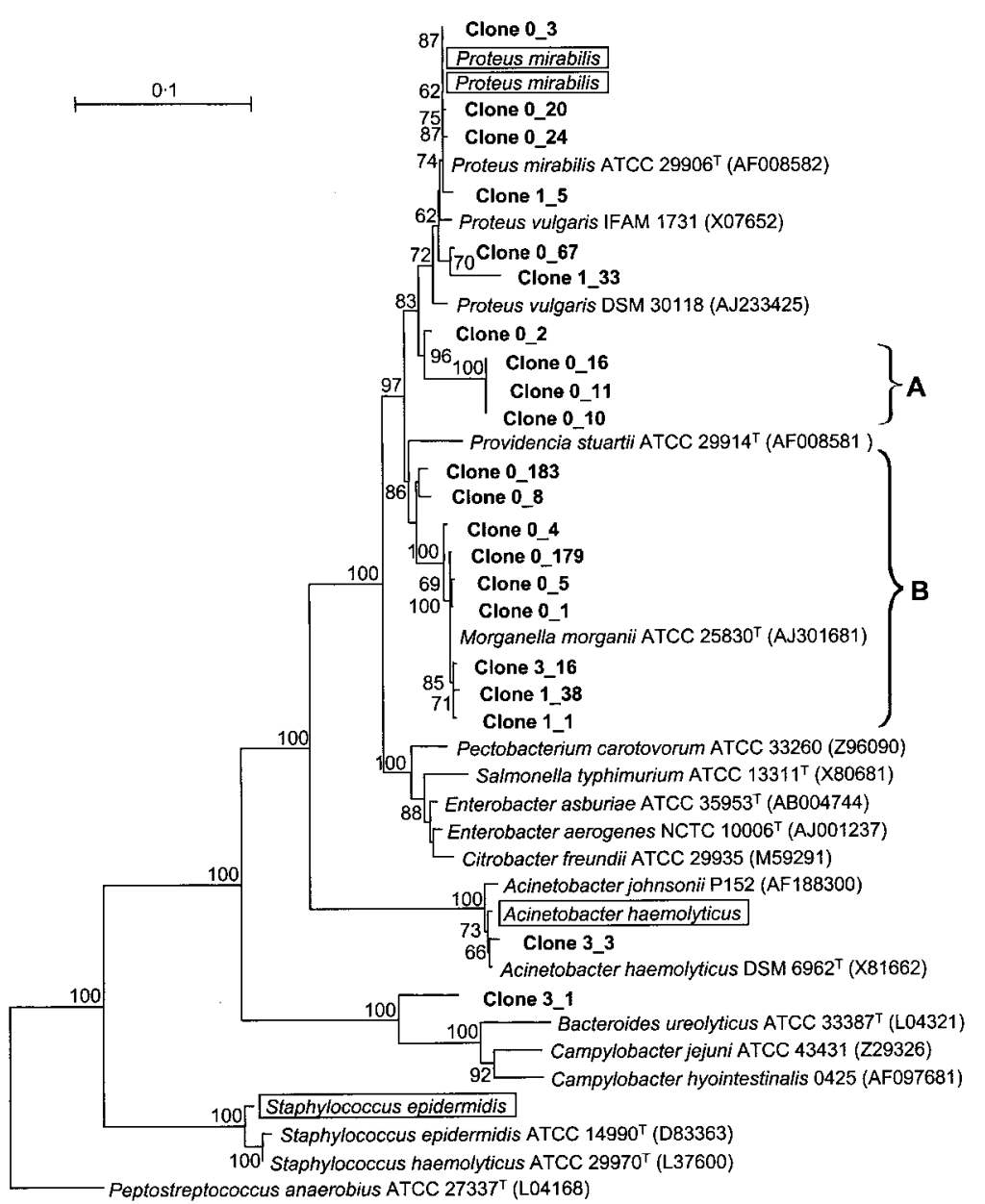

Fig. 1. Phylogenetic tree showing a comparison of sequences from cultured and uncultured micro-organisms from a CVLU wound. Key to primers used: 0_*, 63f/1387r; 1_*, 27f/1492r; $3_{-}^{*}, 27 f / 1525 r$. Boxed sequences are from organisms cultured from the wound. Bootstrap values from 1000 replicate trees are shown at nodes. The scale bar represents $10 \%$ sequence divergence. All other sequences are from the GenBank/EMBL databases (accession numbers given). known sequence, a level of variation that is often found between members of different genera. None of the cultivated sequences exhibited this degree of sequence dissimilarity. Significantly, three clones, 3_1, 5_63 and 5_69, showed very low similarity $(<90 \cdot 2 \%)$ to all database sequences (most similar sequences from Bacteroides ureolyticus, Lactosphaera pasteurii and Acinetobacter lwoffi, respectively) and were not detected by culture and may therefore represent novel, previously uncultured species (Fig. 1; Table 2). In addition, nine clones in cluster B (Fig. 1) and clone 5_75 (Table 2) appeared to have high similarity, $>95 \%$ (clones $0 \_183,0 \_8$ ) and $>98 \%$ (clones $0 \_4$ to $1 \_1$ ), to a sequence from Morganella morganii ATCC $25830^{\mathrm{T}}$, again a species that was not cultured from this wound.

\section{DISCUSSION}

All previous studies to enumerate and identify the microflora of venous leg ulcers have been conducted by bacterial culture alone (Bowler, 1998). This is, to our knowledge, the first such

Table 2. 16S rRNA sequencing of five clones amplified using Gram-positivebacteria-specific primers

\begin{tabular}{|lrlc|}
\hline Clone & Primers & 16S rRNA species identification & $\begin{array}{c}\text { Sequence } \\
\text { identity (\%) }\end{array}$ \\
\hline 4_38 & $712 \mathrm{f} / 1067 \mathrm{r}$ & Enterococcus faecalis & $99 \cdot 1$ \\
5_60 & $1185 \mathrm{f} / 1540 \mathrm{r}$ & Peptostreptococcus octavius & 97 \\
$5 \_75$ & $1185 \mathrm{f} / 1540 \mathrm{r}$ & Morganella morganii & 95 \\
5_69 & $1185 \mathrm{f} / 1540 \mathrm{r}$ & Acinetobacter lwoffi & $89 \cdot 8^{\star}$ \\
5_63 & $1185 \mathrm{f} / 1540 \mathrm{r}$ & Lactosphaera pasteurii & $89 \cdot 3^{*}$ \\
\hline
\end{tabular}

*Very low identity to database sequences indicates that these clones may represent novel phylotypes. 
study applying both a cultural and a molecular approach to chronic wounds. Indeed, few medically important microbial communities have been studied extensively in this way. The microflora associated with the oral cavity, including the human subgingival crevice (Kroes et al., 1999), endodontic infections (Rolph et al., 2001) and childhood caries (Becker et al., 2002), has been studied. Importantly, all of these studies have shown the importance of the molecular approach in revealing greater species diversity. Similarly, our data suggest that cultivation alone underrepresents the true extent of bacterial diversity within the chronic wound and that a more comprehensive analysis is possible with the application of molecular techniques (Hugenholz \& Pace, 1996). A number of clones closely related to cultured organisms such as Acinetobacter haemolyticus and Proteus mirabilis were detected. More importantly, however, clones most closely related to Morganella morganii, the anaerobe Bacteroides ureolyticus and the Gram-positive species Enterococcus faecalis, Peptostreptococcus octavius and Lactosphaera pasteurii were amplified directly from tissue, although these species had not been cultured from this wound.

Proteus spp. were cultured only from the swab, perhaps indicating that it was only a surface colonizer, although, conversely, clones similar to Proteus spp. were detected by molecular means from the tissue. Notably, we have observed little variation in sampling within a biopsy specimen when it was divided into quarters prior to cultural analysis, isolating identical bacterial species and at similar bacterial counts from each biopsy segment (unpublished observations). It is therefore unclear why we were unable to detect Proteus in the tissue by cultural means, although it is possible that cells able to invade the deep tissue may change their phenotype to a semi-dormant state (viable but not culturable), making them difficult to revive by standard culture. Staphylococcus epidermidis was not detected in the swab, presumably as it had colonized the deep tissues and not the wound surface. Surprisingly, Staphylococcus spp. were not amplified by PCR, although clearly present in the tissue from the cultural analysis.

Along with other researchers, we have highlighted the importance of analysis with multiple primer sets in order to obtain the widest spectrum of phylogenetic groups and to reduce the effect of primer biases in such studies (Kroes et al., 1999; Paster et al., 2001). All primers used by us and others have been validated extensively against a wide range of bacteria yet, despite this, biases are apparent. It is possible that the primers behave differently, perhaps annealing less efficiently, when applied in mixed culture. Certainly, in this study, despite the validated universal nature of a number of the primers employed, quite different species were detected depending on the primer set used. Moreover, the Grampositive-specific primers were shown to be capable of detecting sequences representative of Gram-negative species from this wound. This selective amplification reflects a pitfall in the PCR, where primer annealing of one DNA target can be biased, regardless of the initial proportions of the templates (Suzuki \& Giovannoni, 1996). Notwithstanding these biases, however, it is apparent that greater understanding of these biases is needed and that the molecular approach is essential to begin to reveal the true extent of bacterial diversity.

Clones 3_1 and 0_16 to $0 \_10$ (as they appear in the dendrogram in Fig. 1) are most interesting, in that they represent novel phylotypes, a phylotype being defined as a cluster that differs from known species by $\sim 2 \%$ and where members are at least $99 \%$ similar to other members of the same cluster (Paster et al., 2001). Importantly, phylotypes represented only by clones (e.g. clones 3_1, 5_63 and 5_69) may represent currently uncultured or unrecognized potential pathogens. Sequences from the uncultured organisms can be used to design PCR primers for subsequent application to larger numbers of clinical samples, for example via DNA microarrays or using quantitative PCR, hence facilitating the identification of potential markers of disease. Ten clones were found to be most closely related to Morganella morganii (clones 5_75 and 0_16 to 0_10), which is amenable to culture and has previously been detected in chronic wounds (Bowler \& Davies, 1999), but was not cultured in this study. Hence, there appears to be a bias in the cultural analysis. It is possible that less numerous or less vigorous species may have been swamped by the predominant ones and, hence, that they were out-competed on the nonselective media used for culture. One species commonly found in venous ulceration, Pseudomonas aeruginosa, was not detected in this patient.

The debate still exists as to whether it is relevant to treat venous leg ulcers routinely with antibiotic (topical and systemic) or antiseptic (topical) therapies. Clearly, the patient in this study had a colonized ulcer, but had no apparent signs of clinical infection, so it is debatable whether they would have benefited in the long term from such treatment. Antibiotic therapy (usually broad-spectrum) is effective in reducing the bacterial burden of such wounds in the short term, i.e. for the duration of the antibiotic application. However, once stopped, the microflora is quickly able to reestablish itself. This means that routine microbiological sampling of such wounds is therefore often pointless. Clearly, appropriate systemic antibiotics are essential for the treatment of deteriorating, clinically infected wounds characterized by severe pain, oedema, erythema and strong odour. In such cases, microbiological sampling of the wound can be an important aid to appropriate antibiotic therapy.

This study demonstrates that molecular techniques can detect the presence of bacteria in chronic wounds when culture techniques yield a negative result and can be used to identify a wider range of chronic wound-related bacteria, including the presence of previously unidentified or unculturable bacteria. The clinical relevance of the organisms detected in this study remains unclear. However, the comprehensive characterization of the microflora in this way is a prerequisite for the elucidation of associations between bacterial carriage, clinical outcome and effective treatment of this important condition. 


\section{ACKNOWLEDGEMENTS}

We are extremely grateful to Research into Ageing (grant no. 196) for support for K. E. H. We also thank Andy Gane and Gareth Lewis for sequencing and Dr Joanna Hilton and the Wound Healing Research Unit, University Hospital of Wales, for providing the clinical information and specimen.

\section{REFERENCES}

Becker, M. R., Paster, B. J., Leys, E. J., Moeschberger, M. L., Kenyon, S. G., Galvin, J. L., Boches, S. K., Dewhirst, F. E. \& Griffen, A. L. (2002). Molecular analysis of bacterial species associated with childhood caries. J Clin Microbiol 40, 1001-1009.

Bowden, G. H. \& Hardie, J. M. (1971). Anaerobic organisms from the mouth. In Isolation of Anaerobes, pp. 177-205. Edited by D. A. Shapton \& R. G. Board. London: Academic Press.

Bowler, P. G. (1998). The anaerobic and aerobic microbiology of wounds: a review. Wounds 10, 170-178.

Bowler, P. G. \& Davies, B. J. (1999). The microbiology of acute and chronic wounds. Wounds 11, 72-78.

Carroll, N. M., Jaeger, E. E., Choudhury, S., Dunlop, A. A., Matheson, M. M., Adamson, P., Okhravi, N. \& Lightman, S. (2000). Detection of and discrimination between gram-positive and gram-negative bacteria in intraocular samples by using nested PCR. J Clin Microbiol 38, 1753-1757.

Dymock, D., Weightman, A. J., Scully, C. \& Wade, W. G. (1996). Molecular analysis of microflora associated with dentoalveolar abscesses. J Clin Microbiol 34, 537-542.

Falanga, V. (1993). Chronic wounds: pathophysiologic and experimental considerations. J Invest Dermatol 100, 721-725.

Halbert, A. R., Stacey, M. C., Rohr, J. B. \& Jopp-McKay, A. (1992). The effect of bacterial colonization on venous ulcer healing. Australas $J$ Dermatol 33, 75-80.

Hansson, C., Hoborn, J., Moller, A. \& Swanbeck, G. (1995). The microbial flora in venous leg ulcers without clinical signs of infection. Repeated culture using a validated standardised microbiological technique. Acta Derm Venereol 75, 24-30.

Hill, K. E., Marchesi, J. R. \& Weightman, A. J. (1999). Investigation of two evolutionarily unrelated halocarboxylic acid dehalogenase gene families. J Bacteriol 181, 2535-2547.
Hugenholz, P. \& Pace, N. R. (1996). Identifying microbial diversity in the natural environment: a molecular phylogenetic approach. Trends Biotechnol 14, 190-197.

Keay, S., Zhang, C.-O., Baldwin, B. R., Alexander, R. B. \& Warren, J. W. (1998). Polymerase chain reaction amplification of bacterial $16 \mathrm{~S}$ rRNA genes from cold-cup biopsy forceps. J Urol 160, 2229-2231.

Klausegger, A., Hell, M., Berger, A., Zinober, K., Baier, S., Jones, N., Sperl, W. \& Kofler, B. (1999). Gram type-specific broad-range PCR amplification for rapid detection of 62 pathogenic bacteria. J Clin Microbiol 37, 464-466.

Kroes, I., Lepp, P. W. \& Relman, D. A. (1999). Bacterial diversity within the human subgingival crevice. Proc Natl Acad Sci U S A 96, 14547-14552.

Lane, D. J. (1991). 16S/23S rRNA sequencing. In Nucleic Acid Techniques in Bacterial Systematics, pp. 115-175. Edited by E. Stackebrandt \& M. Goodfellow. London: Wiley.

Marchesi, J. R., Sato, T., Weightman, A. J., Martin, T. A., Fry, J. C., Hiom, S. J., Dymock, D. \& Wade, W. G. (1998). Design and evaluation of useful bacterium-specific PCR primers that amplify genes coding for bacterial 16S rRNA. Appl Environ Microbiol 64, 795-799.

Murdoch, D. A., Mitchelmore, I. J. \& Tabaqchali, S. (1994). The clinical importance of gram-positive anaerobic cocci isolated at St Bartholomew's Hospital, London, in 1987. J Med Microbiol 41, 36-44.

Paster, B. J., Boches, S. K., Galvin, J. L., Ericson, R. E., Lau, C. N., Levanos, V. A., Sahasrabudhe, A. \& Dewhirst, F. E. (2001). Bacterial diversity in human subgingival plaque. J Bacteriol 183, 3770-3783.

Rolph, H. J., Lennon, A., Riggio, M. P., Saunders, W. P., MacKenzie, D., Coldero, L. \& Bagg, J. (2001). Molecular identification of microorganisms from endodontic infections. J Clin Microbiol 39, 3282-3289.

Sambrook, J., Fritsch, E. F. \& Maniatis, T. (1989). Molecular Cloning: $a$ Laboratory Manual, 2nd edn. Cold Spring Harbor, NY: Cold Spring Harbor Laboratory.

Suzuki, M. T. \& Giovannoni, S. J. (1996). Bias caused by template annealing in the amplification of mixtures of $16 \mathrm{~S}$ rRNA genes by PCR. Appl Environ Microbiol 62, 625-630.

Wilson, M. J., Weightman, A. J. \& Wade, W. G. (1997). Applications of molecular ecology in the characterisation of uncultured microorganisms associated with human disease. Rev Med Microbiol 8, 91-101. 